\title{
Application of wavefront coordinates to acoustic ray tracing
}

\author{
John P. Best* \\ (Received 20 September 1996)
}

\begin{abstract}
Acoustic wave propagation is considered by transforming the equations of inviscid compressible flow to a coordinate system defined by the wavefront geometry. These equations are linearised and equations for the trajectory of rays are derived in the high frequency limit. The formulation in terms of the new coordinates facilitates a rapid derivation of an expression for the
\end{abstract}

* Maritime Operations Division, Aeronautical and Maritime Research Laboratory, Defence Science and Technology Organisation, PO Box 4331, Melbourne, Victoria 3001, Australia. mailto:jpbestetaz.dra.hmg.gb

See http://jamsb.sci.usq.edu.au/V39/E001/home.html for this paper and ancillary services, (c) Austral. Mathematical Soc. 1997. Published 21 Oct 1997, last updated October 21, 1997. 
transmission loss associated with propagation along a ray. The form of the equations permits easy and robust calculation of sound propagation through media characterised by a non-uniform sound speed and demonstrates the utility of the coordinate system defined by the natural geometry of the wavefront.

\section{Contents}

1 Introduction

2 The wavefront coordinate system

3 Derivation of ray trace equations

4 Discussion

A Appendix

\section{Introduction}

Ray tracing is a technique widely applied to approximately calculate wave propagation in acoustics and optics. In addition to predicting the trajectory followed 
by rays, often normal to the wavefront, a critical quantity required is the variation of wave intensity along a ray. The approach usually used is to supplement the equations defining the trajectory of a ray with additional equations that describe the variation of the intensity, or transmission loss as it is known in underwater acoustics. This approach has been considered by Krol [4], Solomon \& Armijo [5] and Uginičius $[6,7]$. A very general formulation for dispersive inhomogeneous media has been given by Buckley [2] and a recent exposition in the context of electromagnetic propagation in the ionosphere is that of Budden [3].

In this paper, an alternative derivation of the ray trace equations is given, along with an expression for the variation of intensity along a ray. The feature that differentiates this work from that carried out previously is that the derivation here proceeds by introducing a coordinate system defined by the wavefront geometry, hence the terminology wavefront coordinates. The equations of inviscid compressible flow are transformed to this new coordinate system and the ray trace equations derived. The equations deduced are readily implemented in a robust numerical scheme in order that propagation may be considered in non-homogeneous media. Calculation of the wave intensity along a ray is particularly straight forward in this formalism and demonstrates the utility of the coordinate system that may be considered natural for this problem. 


\section{The wavefront coordinate system}

Consider the equations of inviscid gas dynamics in three dimensions. They may be written as

$$
\begin{gathered}
\partial_{t} \rho+\partial_{x}(\rho u)+\partial_{y}(\rho v)+\partial_{z}(\rho w)=0 \\
\partial_{t} u+u \partial_{x} u+v \partial_{y} u+w \partial_{z} u+\partial_{x} p / \rho=0 \\
\partial_{t} v+u \partial_{x} v+v \partial_{y} v+w \partial_{z} v+\partial_{y} p / \rho=0 \\
\partial_{t} w+u \partial_{x} w+v \partial_{y} w+w \partial_{z} w+\partial_{z} p / \rho=0, \\
\partial_{t} p+u \partial_{x} p+v \partial_{y} p+w \partial_{z} p-a^{2}\left(\partial_{t} \rho+u \partial_{x} \rho+v \partial_{y} \rho+w \partial_{z} \rho\right)=0,
\end{gathered}
$$

where

$$
a^{2}=(\partial p / \partial \rho)_{S},
$$

with $S$ the entropy. In the above equations $\rho$ is the fluid density, $p$ is the pressure, $(u, v, w)$ is the fluid velocity in Cartesian coordinates $(x, y, z)$ and $a$ is the sound speed. $\partial_{\mu}$ denotes a partial derivative with respect to $\mu$. In the case where the fluid is an ideal gas

$$
a^{2}=\gamma p / \rho
$$

where $\gamma$ is the ratio of specific heats.

This paper is concerned with small amplitude wave propagation described by the linearised form of (1)-(6), although it is noted that problems involving the 
propagation of shock waves may be dealt with using similar ideas to those presented here [1]. At any point on the wavefront the normal vector is $\boldsymbol{I}$, given in terms of angles $\theta$ and $\phi$ as

$$
\boldsymbol{I}=(\cos \phi \cos \theta, \sin \phi \cos \theta, \sin \theta) .
$$

Consider a local orthonormal coordinate system defined at a point by the direction of $\boldsymbol{I}$ when the wavefront passes this point. The coordinate in the direction of propagation is $\eta$ and the other two coordinate directions are $\zeta$ and $\xi$. As a matter of convention $\zeta$ is chosen to lie in the $x y$ plane. This coordinate system is illustrated in Figure 1. The coordinate system is defined formally by its relation to the Cartesian coordinate system;

$$
\begin{aligned}
d \eta & =\cos \phi \cos \theta d x+\sin \phi \cos \theta d y+\sin \theta d z, \\
d \zeta & =-\sin \phi d x+\cos \phi d y, \\
d \xi & =-\cos \phi \sin \theta d x-\sin \phi \sin \theta d y+\cos \theta d z,
\end{aligned}
$$

from which it follows that

$$
\begin{aligned}
& \partial_{x}=\cos \phi \cos \theta \partial_{\eta}-\sin \phi \partial_{\zeta}-\cos \phi \sin \theta \partial_{\xi}, \\
& \partial_{y}=\sin \phi \cos \theta \partial_{\eta}+\cos \phi \partial_{\zeta}-\sin \phi \sin \theta \partial_{\xi}, \\
& \partial_{z}=\sin \theta \partial_{\eta}+\cos \theta \partial_{\xi} .
\end{aligned}
$$

It is also useful to note that

$$
\begin{aligned}
\partial_{\eta} & =\cos \phi \cos \theta \partial_{x}+\sin \phi \cos \theta \partial_{y}+\sin \theta \partial_{z}, \\
\partial_{\zeta} & =-\sin \phi \partial_{x}+\cos \phi \partial_{y}, \\
\partial_{\xi} & =-\cos \phi \sin \theta \partial_{x}-\sin \phi \sin \theta \partial_{y}+\cos \theta \partial_{z} .
\end{aligned}
$$


For future convenience the commutator notation $[A, B]=A B-B A$ is introduced. Noting that $\left[\partial_{x}, \partial_{y}\right]=\left[\partial_{y}, \partial_{z}\right]=\left[\partial_{z}, \partial_{x}\right]=0$ for functions with continuous second derivatives it follows using (10) that

$$
\begin{aligned}
& {\left[\partial_{\eta}, \partial_{\zeta}\right]=-\cos \theta \partial_{\eta} \phi \partial_{\eta}-\cos \theta \partial_{\zeta} \phi \partial_{\zeta}+\left(\sin \theta \partial_{\eta} \phi-\partial_{\zeta} \theta\right) \partial_{\xi},} \\
& {\left[\partial_{\zeta}, \partial_{\xi}\right]=\left(\cos \theta \partial_{\xi} \phi-\partial_{\zeta} \theta\right) \partial_{\eta}-\sin \theta \partial_{\zeta} \phi \partial_{\zeta}-\sin \theta \partial_{\xi} \phi \partial_{\xi},} \\
& {\left[\partial_{\xi}, \partial_{\eta}\right]=\partial_{\eta} \theta \partial_{\eta}+\left(\sin \theta \partial_{\eta} \phi+\cos \theta \partial_{\xi} \phi\right) \partial_{\zeta}+\partial_{\xi} \theta \partial_{\xi},}
\end{aligned}
$$

so that partial derivatives in $\eta, \zeta$ and $\xi$ do not commute.

Before proceeding it is pertinent to consider in a little more detail the transformation to the new coordinate system. Firstly consider a wavefront area element $A$ at the point $(\eta, \zeta, \xi)$ as illustrated in Figure 2. It is given by

$$
A(\eta, \zeta, \xi)=d \xi d \zeta
$$

At $(\eta, \zeta, \xi+d \xi)$ the direction of propagation is to leading order defined by $\theta+$ $\partial_{\xi} \theta d \xi, \phi+\partial_{\xi} \phi d \xi$ and at $(\eta, \zeta+d \zeta, \xi)$ by $\theta+\partial_{\zeta} \theta d \zeta, \phi+\partial_{\zeta} \phi d \zeta$. Hence at $(\eta+d \eta, \zeta, \xi)$ this element of the wavefront has, to leading order, sides of length $d \xi+\partial_{\xi} \theta d \xi d \eta$ and $d \zeta+\cos \theta \partial_{\zeta} \phi d \zeta d \eta$. Hence the area element is

$$
A(\eta+d \eta, \zeta, \xi)=A(\eta, \zeta, \xi)\left(1+\left(\partial_{\xi} \theta+\cos \theta \partial_{\zeta} \phi\right) d \eta+O\left(d \eta^{2}\right)\right),
$$

from which it is deduced that

$$
\partial_{\eta} A / A=\partial_{\xi} \theta+\cos \theta \partial_{\zeta} \phi .
$$




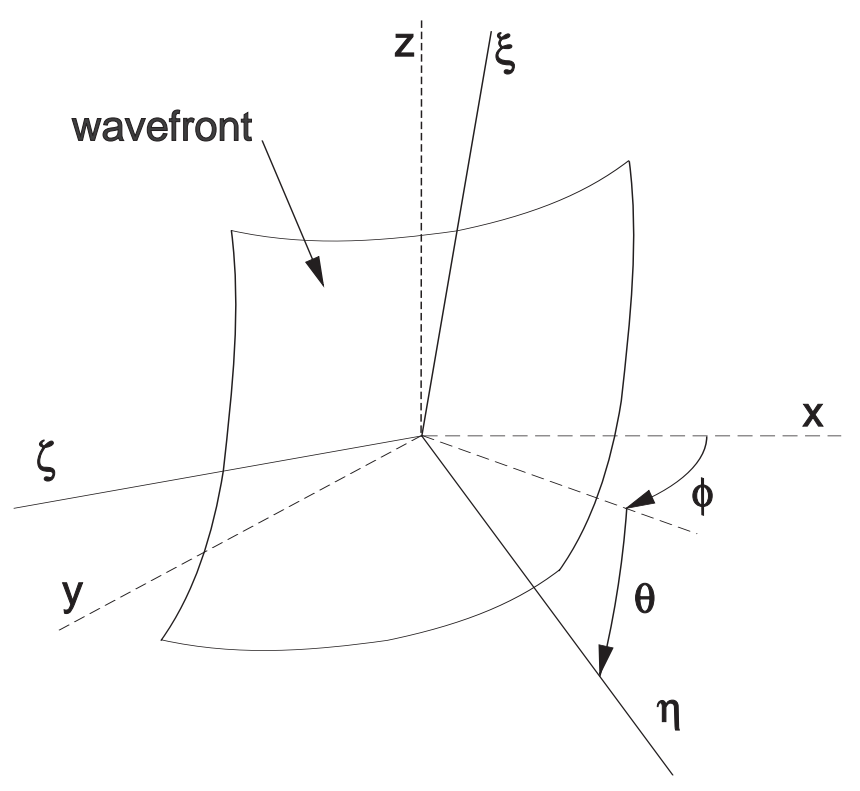

FIGURE 1. The wavefront coordinate system. 


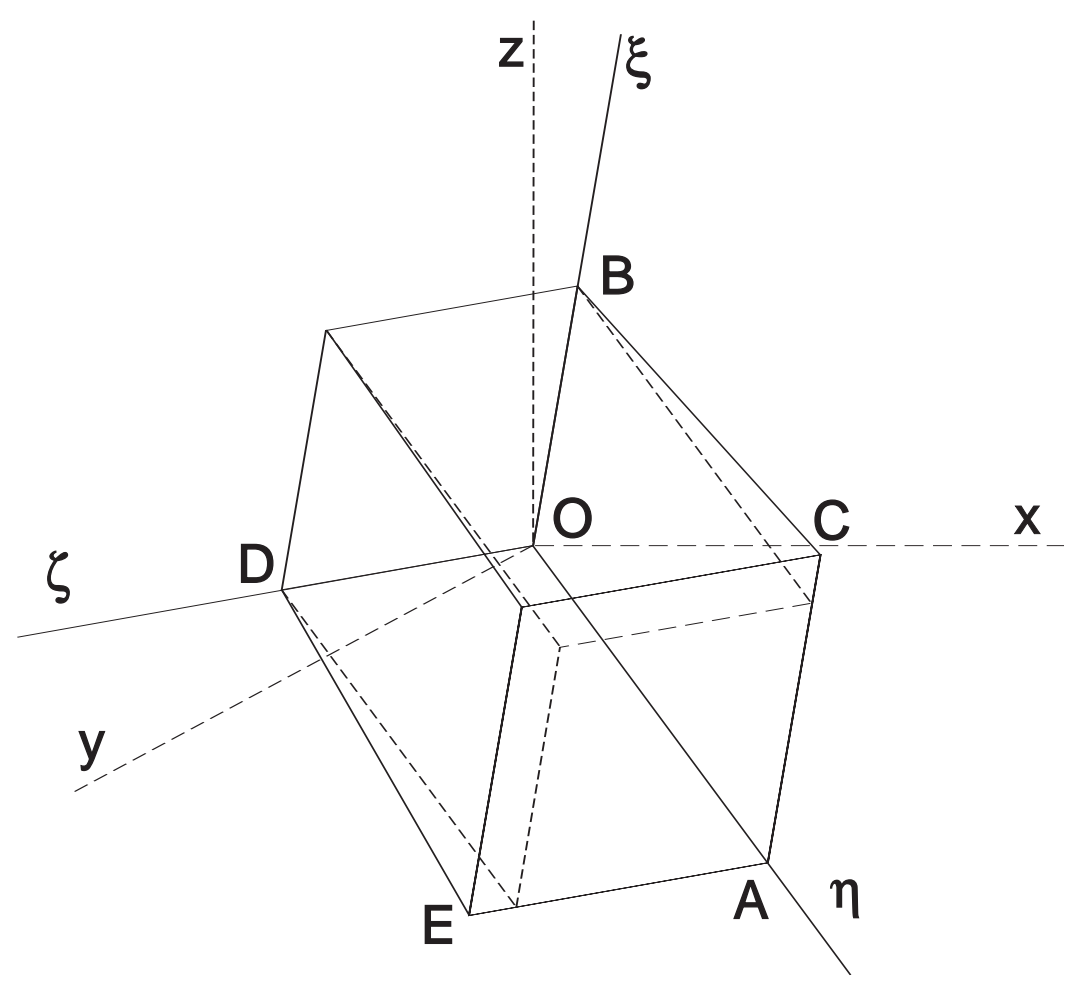

FIGURE 2. Propagation of a wavefront area element. 
Since the intensity of a wave is fundamentally dependent upon the wavefront area it is expected that this quantity will assume significance in derivation of an equation for the variation of the intensity along a ray. Notice that the variation of the area is dependent upon the local geometry of the wavefront. In particular, the rate of change of the area is dependent upon the spatial rate of change of the direction of propagation across the wavefront.

Next consider the manner in which the angles $\theta$ and $\phi$ change along a ray. Let the normal speed of the wavefront be $c$. This is not necessarily equal to the ambient sound speed $a_{0}$. Consider the point $\mathbf{B}$ in Figure 3 with coordinates $(\eta, \zeta, \xi+d \xi)$. The normal speed of propagation is $c+\partial_{\xi} c d \xi$, so it follows that $\mathbf{B}^{\prime} \mathbf{C}=\partial_{\xi} c d \xi d \eta / c$ so

$$
d \theta=-\partial_{\xi} c d \eta / c
$$

from which it is deduced

$$
\partial_{\eta} \theta=-\partial_{\xi} c / c
$$

Similar consideration of the point $\mathbf{D}$ in Figure 4 with coordinates $(\eta, \zeta+d \zeta, \xi)$ yields

$$
\partial_{\eta} \phi=-\partial_{\zeta} c /(c \cos \theta) .
$$

To proceed, (1) is transformed to the new coordinate system and in doing so fluid velocity components $(\bar{u}, \bar{v}, \bar{w})$ are defined with respect to the new coordinates and are related to the original components by

$$
\begin{aligned}
u & =\cos \phi \cos \theta \bar{u}-\sin \phi \bar{v}-\cos \phi \sin \theta \bar{w}, \\
v & =\sin \phi \cos \theta \bar{u}+\cos \phi \bar{v}-\sin \phi \sin \theta \bar{w}, \\
w & =\sin \theta \bar{u}+\cos \theta \bar{w} .
\end{aligned}
$$




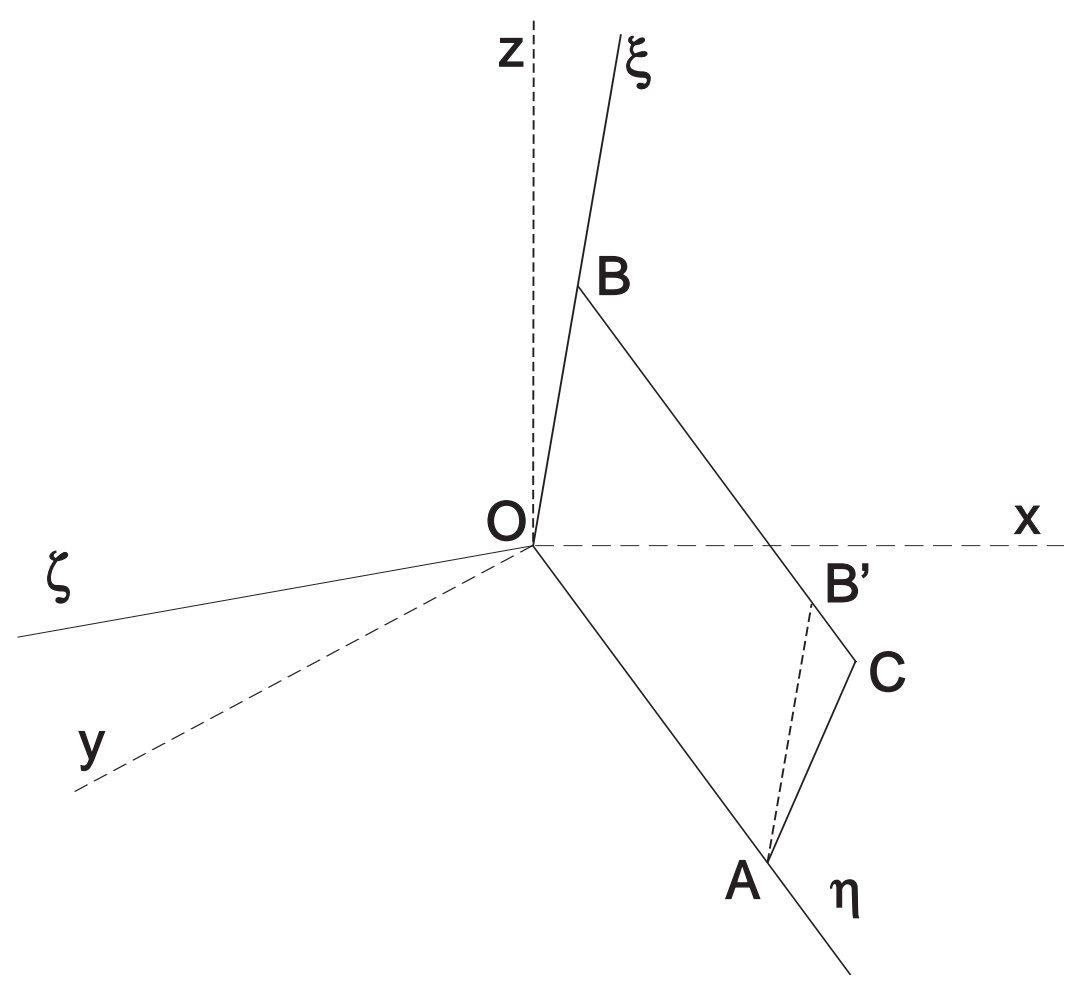

FIgURE 3 . The change in $\theta$ along a ray. 


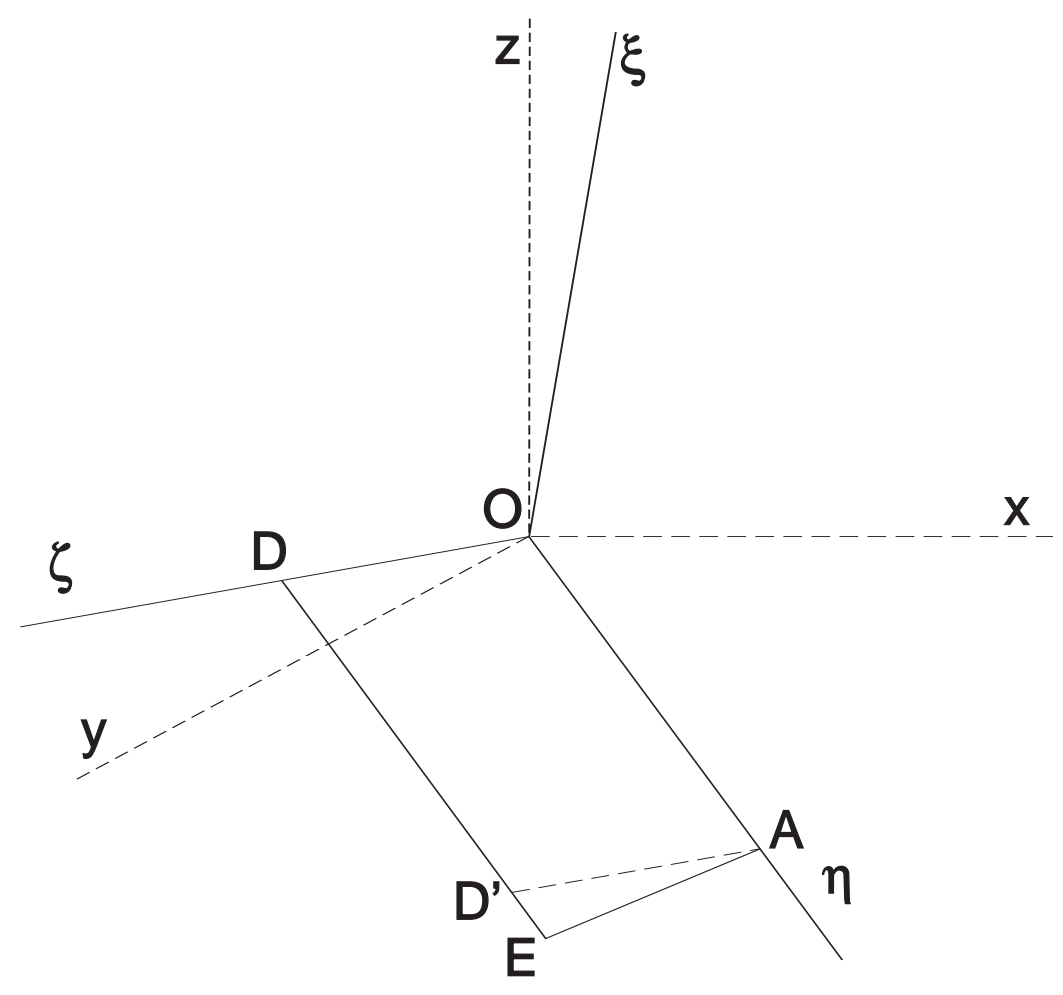

FIGURE 4. The change in $\phi$ along a ray. 
It may be readily shown that

$$
u \partial_{x}+v \partial_{y}+w \partial_{z}=\bar{u} \partial_{\eta}+\bar{v} \partial_{\zeta}+\bar{w} \partial_{\xi}
$$

Using (10), (19) and (20) in (1) and dropping the bar over velocity components yields

$$
\begin{gathered}
\partial_{t} \rho+\partial_{\eta}(\rho u)+\partial_{\zeta}(\rho v)+\partial_{\xi}(\rho w)-\rho w \partial_{\eta} \theta+\rho u \partial_{\xi} \theta-\rho v \cos \theta \partial_{\eta} \phi \\
+\rho(u \cos \theta-w \sin \theta) \partial_{\zeta} \phi+\rho v \sin \theta \partial_{\xi} \phi=0 \\
\partial_{t} u+u \partial_{\eta} u+v \partial_{\zeta} u+w \partial_{\xi} u+\partial_{\eta} p / \rho-u w \partial_{\eta} \theta-v w \partial_{\zeta} \theta-w^{2} \partial_{\xi} \theta \\
-u v \cos \theta \partial_{\eta} \phi-v^{2} \cos \theta \partial_{\zeta} \phi-v w \cos \theta \partial_{\xi} \phi=0 \\
\partial_{t} v+u \partial_{\eta} v+v \partial_{\zeta} v+w \partial_{\xi} v+\partial_{\zeta} p / \rho \\
+(u \cos \theta-w \sin \theta)\left(u \partial_{\eta} \phi+v \partial_{\zeta} \phi+w \partial_{\zeta} \phi\right)=0, \\
\partial_{t} w+u \partial_{\eta} w+v \partial_{\zeta} w+w \partial_{\xi} w+\partial_{\xi} p / \rho+u^{2} \partial_{\eta} \theta+u v \partial_{\zeta} \theta+u w \partial_{\xi} \theta \\
+u v \sin \theta \partial_{\eta} \phi+v^{2} \sin \theta \partial_{\zeta} \phi+v w \sin \theta \partial_{\xi} \phi=0 \\
\partial_{t} p+u \partial_{\eta} p+v \partial_{\zeta} p+w \partial_{\xi} p-a^{2}\left(\partial_{t} \rho+u \partial_{\eta} \rho+v \partial_{\zeta} \rho+w \partial_{\xi} \rho\right)=0 .
\end{gathered}
$$

The consideration of small amplitude wave propagation proceeds by linearising (21)-(25) about undisturbed values of the flow variables. The undisturbed density, pressure and sound speed are denoted by $\rho_{0}, p_{0}$ and $a_{0}$ and hereafter $\rho$ and $p$ refer to the perturbations in density and pressure caused by the wave motion. In the following derivation it is assumed that $p_{0}$ is uniform and that $\rho_{0}$ and $a_{0}$ may 
depend upon the spatial coordinates, so that the propagation medium may be nonhomogeneous. The undisturbed fluid velocity is considered to be equal to zero. Performing the linearisation yields

$$
\begin{gathered}
\partial_{t} \rho+\rho_{0}\left(\partial_{\eta} u+\partial_{\zeta} v+\partial_{\xi} w\right)+u \partial_{\eta} \rho_{0}+v \partial_{\zeta} \rho_{0}+w \partial_{\xi} \rho_{0}-\rho_{0} w \partial_{\eta} \theta+\rho_{0} u \partial_{\xi} \theta \\
-\rho_{0} v \cos \theta \partial_{\eta} \phi+\rho_{0}(u \cos \theta-w \sin \theta) \partial_{\zeta} \phi+\rho_{0} v \sin \theta \partial_{\xi} \phi=0 \\
\partial_{t} u+\partial_{\eta} p / \rho_{0}=0, \quad \partial_{t} v+\partial_{\zeta} p / \rho_{0}=0, \quad \partial_{t} w+\partial_{\xi} p / \rho_{0}=0 \\
\partial_{t} p-a_{0}^{2}\left(\partial_{t} \rho+u \partial_{\eta} \rho_{0}+v \partial_{\zeta} \rho_{0}+w \partial_{\xi} \rho_{0}\right)=0
\end{gathered}
$$

An alternative derivation may have proceeded by linearising (1)-(5) and then undertaking the transformation to the $(\eta, \zeta, \xi)$ coordinate system. This process yields exactly (26)-(28).

\section{Derivation of ray trace equations}

Eliminating the velocity components and density from (26)-(28) yields

$$
\begin{aligned}
& \partial_{t}^{2} p / a_{0}^{2}-\left(\partial_{\eta}^{2} p+\partial_{\zeta}^{2} p+\partial_{\xi}^{2} p\right)+\left(\partial_{\eta} \rho_{0} \partial_{\eta} p+\partial_{\zeta} \rho_{0} \partial_{\zeta} p+\partial_{\xi} \rho_{0} \partial_{\xi} p\right) / \rho_{0}+\partial_{\xi} p \partial_{\eta} \theta \\
& -\partial_{\eta} p \partial_{\xi} \theta+\cos \theta \partial_{\zeta} p \partial_{\eta} \phi-\left(\cos \theta \partial_{\eta} p-\sin \theta \partial_{\xi} p\right) \partial_{\zeta} \phi-\sin \theta \partial_{\zeta} p \partial_{\xi} \phi=0
\end{aligned}
$$


Following Whitham [8], assume an asymptotic solution of the form

$$
\begin{aligned}
p & =e^{-i \omega(t-\sigma(\mathbf{r}))} \sum_{n=0}^{\infty} P_{n}(\mathbf{r})(-i \omega)^{-n} \\
& =\sum_{n=0}^{\infty} P_{n}(\mathbf{r}) f_{n}(S)
\end{aligned}
$$

where

$$
S=t-\sigma(\mathbf{r}), \quad f_{n}(S)=\frac{e^{-i \omega S}}{(-i \omega)^{n}}
$$

Notice that

$$
f_{n}^{\prime}(S)=f_{n-1}(S) .
$$

The symbol $\mathbf{r}$ denotes some point in space whose position may be specified by either Cartesian coordinates or $(\eta, \zeta, \xi)$ coordinates. The form of this asymptotic solution is applicable in the high frequency limit and the surfaces $S=$ constant denote surfaces of constant phase, otherwise known as wavefronts. With an appropriate choice of the functions $f_{n}$, this form of solution is equally applicable to the case of propagation of a surface of discontinuity [8].

Recall that the coordinates $(\eta, \zeta, \xi)$ are defined in terms of the normal to the wavefront. Hence it is evident that since $\sigma$ is constant over a wavefront, it follows 
that $\partial_{\zeta}^{n} \sigma \equiv 0$ and $\partial_{\xi}^{n} \equiv 0$ for $n \geq 1$. Using (30) in (29) yields

$$
\begin{aligned}
&\left.\sum_{n=0}^{\infty}\left\{\begin{array}{l}
{[} \\
{[}
\end{array}\right) a_{0}^{2}-\left(\partial_{\eta} \sigma\right)^{2}\right] P_{n} f_{n-2} \\
&+\left[2 \partial_{\eta} \sigma \partial_{\eta} P_{n}+\left(\partial_{\eta}^{2} \sigma-\partial_{\eta} \sigma \partial_{\eta} \rho_{0} / \rho_{0}\right) P_{n}+\left(\partial_{\xi} \theta+\cos \theta \partial_{\zeta} \phi\right) \partial_{\eta} \sigma P_{n}\right] f_{n-1} \\
&-\left[\partial_{\eta}^{2} P_{n}+\partial_{\zeta}^{2} P_{n}+\partial_{\xi}^{2} P_{n}-\left(\partial_{\eta} \rho_{0} \partial_{\eta} P_{n}+\partial_{\zeta} \rho_{0} \partial_{\zeta} P_{n}+\partial_{\xi} \rho_{0} \partial_{\xi} P_{n}\right) / \rho_{0}\right. \\
&+\left(\partial_{\xi} \theta+\cos \theta \partial_{\zeta} \phi\right) \partial_{\eta} P_{n}+\left(\sin \theta \partial_{\xi} \phi-\cos \theta \partial_{\eta} \phi\right) \partial_{\zeta} P_{n} \\
&\left.\left.-\left(\partial_{\eta} \theta+\sin \theta \partial_{\zeta} \phi\right) \partial_{\xi} P_{n}\right] f_{n}\right\}=0
\end{aligned}
$$

The quantities $\sigma$ and $P_{n}$ are determined by equating coefficients of $f_{n}$ to zero. Equating the coefficients of $f_{-2}$ and $f_{-1}$ to zero yields equations for $\sigma$ and the leading term in the expansion of the wave amplitude $P_{0}$ :

$$
\begin{gathered}
1 / a_{0}^{2}-\left(\partial_{\eta} \sigma\right)^{2}=0, \\
2 \partial_{\eta} \sigma \partial_{\eta} P_{0}+\left(\partial_{\eta}^{2} \sigma-\partial_{\eta} \rho_{0} \partial_{\eta} \sigma / \rho_{0}\right) P_{0}+\left(\partial_{\xi} \theta+\cos \theta \partial_{\zeta} \phi\right) \partial_{\eta} \sigma P_{0}=0,
\end{gathered}
$$

where (34) has been used to eliminate a term involving $P_{1}$ from the coefficient of $f_{-1}$. Equation (34) is the known result that the normal speed of propagation of the wavefront is $a_{0}$. Equation (35) yields the leading order approximation to the wave amplitude and it is from this expression that the variation of the wave intensity along the rays is determined.

Multiplying (35) by $p_{0} / \rho_{0}$ yields

$$
\partial_{\eta}\left(\frac{P_{0}^{2}}{\rho_{0} a_{0}}\right)=-\frac{P_{0}^{2}}{\rho_{0} a_{0}}\left(\partial_{\xi} \theta+\cos \theta \partial_{\zeta} \phi\right)
$$


and identifying $P_{0}^{2} /\left(\rho_{0} a_{0}\right)$ as the intensity $I$ of the sound wave, (36) integrates to

$$
\ln \left(I(\eta) / I\left(\eta_{0}\right)\right)=-\int_{\eta_{0}}^{\eta}\left(\partial_{\xi} \theta+\cos \theta \partial_{\zeta} \phi\right) d \eta .
$$

Recalling (15), which relates the integrand of (37) to the rate of change of the area of an element of the wavefront, it is clear that this relation merely reflects the fact that the energy flux is constant down a tube defined by the normals to the wavefront. It is further remarked that in the traditional approach to geometrical acoustics, rays are defined to be in the direction of the characteristics of the eikonal equation and that these are coincident with the wavefront normal only in the case of an isotropic medium. The rays are in fact in the direction of energy propagation. The preceding analysis shows that energy propagates in the direction defined by $\eta$ and hence the rays are normal to the wavefront. In the event that the medium is non-isotropic, the equation corresponding to (36) has additional terms reflecting the difference between the direction of energy propagation and wavefront normal.

From this point $c$ and $a_{0}$ shall be used interchangeably, since the analysis has demonstrated the known fact that in the case of an isotropic medium the normal speed of the wavefront is equal to the ambient sound speed. The trajectory of the rays is determined as follows. From (11) it follows that

$$
\begin{aligned}
& \partial_{\eta} x=\cos \phi \cos \theta, \\
& \partial_{\eta} y=\sin \phi \cos \theta, \\
& \partial_{\eta} z=\sin \theta .
\end{aligned}
$$

The ray trajectory is determined by integrating these equations simultaneously with (17) and (18). Ordinarily, the sound speed $c$ is known as a function of the 
Cartesian coordinates so converting the derivatives of $c$ in (17) and (18) using (11) yields

$$
\begin{aligned}
\partial_{\eta} \theta & =\cos \phi \sin \theta c_{x} / c+\sin \phi \sin \theta c_{y} / c-\cos \theta c_{z} / c, \\
\partial_{\eta} \phi & =\left(\sin \phi c_{x}-\cos \phi c_{y}\right) /(c \cos \theta) .
\end{aligned}
$$

Thus given the initial direction of a ray and the sound speed distribution, (38) and (39) may be integrated simultaneously to determine the trajectory of the ray. Notice the particularly simple form of these equations.

It is remarked that in the event the sound speed depends in some complex manner on $x$ and $y$ the possibility exists that $\cos \theta=0$ at some point on a ray, rendering the second equation of (39) undefined. If this circumstance is expected the approach to follow is to replace the equation for $\partial_{\eta} \phi$ by equations for $\partial_{\eta}(\cos \phi \cos \theta)$ and $\partial_{\eta}(\sin \phi \cos \theta)$. It may be routinely shown that such equations, in conjunction with (38) and the first equation of (39) yield a closed system, without the possible defect of becoming undefined in the event that $\cos \theta=0$.

The determination of the variation of the intensity, or the transmission loss in underwater acoustics, necessitates that $\partial_{\xi} \theta$ and $\partial_{\zeta} \phi$ be computed along a ray. The approach followed is to obtain expressions for $\partial_{\eta} \partial_{\xi} \theta$ and $\partial_{\eta} \partial_{\zeta} \phi$ and integrate these simultaneously with (38) and (39). This is achieved using the commutator relations (12). Application of these to $\theta$ and $\phi$ yields

$$
\begin{gathered}
\partial_{\eta} \partial_{\xi} \theta=\partial_{\xi} \partial_{\eta} \theta-\left(\partial_{\eta} \theta\right)^{2}-\left(\partial_{\xi} \theta\right)^{2}-\left(\sin \theta \partial_{\eta} \phi+\cos \theta \partial_{\xi} \phi\right) \partial_{\zeta} \theta \\
\partial_{\eta} \partial_{\xi} \phi=\partial_{\xi} \partial_{\eta} \phi-\partial_{\eta} \theta \partial_{\eta} \phi-\partial_{\xi} \theta \partial_{\xi} \phi-\left(\sin \theta \partial_{\eta} \phi+\cos \theta \partial_{\xi} \phi\right) \partial_{\zeta} \phi \\
\partial_{\eta} \partial_{\zeta} \theta=\partial_{\zeta} \partial_{\eta} \theta-\cos \theta \partial_{\eta} \phi \partial_{\eta} \theta-\cos \theta \partial_{\zeta} \phi \partial_{\zeta} \theta+\left(\sin \theta \partial_{\eta} \phi-\partial_{\zeta} \theta\right) \partial_{\xi} \theta
\end{gathered}
$$




$$
\partial_{\eta} \partial_{\zeta} \phi=\partial_{\zeta} \partial_{\eta} \phi-\cos \theta\left(\partial_{\eta} \phi\right)^{2}-\cos \theta\left(\partial_{\zeta} \phi\right)^{2}+\left(\sin \theta \partial_{\eta} \phi-\partial_{\zeta} \theta\right) \partial_{\xi} \phi
$$

In these expressions the terms $\partial_{\xi} \partial_{\eta} \theta, \partial_{\xi} \partial_{\eta} \phi, \partial_{\zeta} \partial_{\eta} \theta$ and $\partial_{\zeta} \partial_{\eta} \phi$ are determined by differentiating (39) and converting derivatives of the sound speed to Cartesian coordinates. The resulting equations are written in full in the appendix. Equations (41) and (42) for $\partial_{\xi} \phi$ and $\partial_{\zeta} \theta$ are given so that (40)-(43) yield a closed system of equations in conjunction with (38) and (39). Despite the number of terms in these expressions it should be noted that in implementing a numerical scheme to solve these equations, such as a Runge-Kutta method, the expressions for the derivatives of the dependent variables merely involve algebraic expressions of these variables, derivatives of the sound speed and elementary trigonometric functions. Their integration is routine. The equation

$$
\partial_{\eta}(\ln I)=-\left(\partial_{\xi} \theta+\cos \theta \partial_{\zeta} \phi\right)
$$

completes the formulation. Simultaneous integration of (44) with the equations describing the ray trajectory permits determination of the intensity at any point on a ray, given the initial ray direction and intensity.

\section{Discussion}

To illustrate the ease with which this formulation may be implemented, the example of underwater sound propagation is considered where the sound velocity is simply a function of the vertical coordinate $z$. Without loss of generality the 
angle $\phi$ is taken to be initially equal to zero. It is further assumed that the initial wavefront is a sphere of radius 1 . Hence it follows that $\partial_{\zeta} \theta$ and $\partial_{\xi} \phi$ are equal to zero here. Suppose that the centre of the sphere is at the origin and the ray under consideration is propagating initially in the $x z$ plane. Thus its original location is $\left(\cos \theta_{i}, 0, \sin \theta_{i}\right)$ where $\theta_{i}$ is the initial value of $\theta$. Equations (38) and (39) become

$$
\begin{aligned}
\partial_{\eta} x & =\cos \theta, \\
\partial_{\eta} y & =0, \\
\partial_{\eta} z & =\sin \theta, \\
\partial_{\eta} \theta & =-\cos \theta c^{\prime} / c, \\
\partial_{\eta} \phi & =0,
\end{aligned}
$$

where $c^{\prime}$ denotes differentiation with respect to $z$. The second and last of these equations show that $y$ and $\phi$ are identically equal to zero, either fact demonstrating that the ray propagates in a vertical plane.

The variation of the intensity is given by (44) and to evaluate the required terms notice that (40)-(43) become

$$
\begin{gathered}
\partial_{\eta} \partial_{\xi} \theta=-\left(\partial_{\eta} \theta\right)^{2}-\left(\partial_{\xi} \theta\right)^{2}-\cos \theta \partial_{\xi} \phi \partial_{\zeta} \theta+c^{\prime} \sin \theta \partial_{\xi} \theta / c \\
-c^{\prime \prime} \cos ^{2} \theta / c+\left(c^{\prime}\right)^{2} \cos ^{2} \theta / c^{2} \\
\partial_{\eta} \partial_{\xi} \phi=-\left(\partial_{\xi} \theta+\cos \theta \partial_{\zeta} \phi\right) \partial_{\xi} \phi \\
\partial_{\eta} \partial_{\zeta} \theta=-\left(\partial_{\xi} \theta+\cos \theta \partial_{\zeta} \phi-c^{\prime} \sin \theta / c\right) \partial_{\zeta} \theta \\
\partial_{\eta} \partial_{\zeta} \phi=-\partial_{\zeta} \theta \partial_{\xi} \phi-\cos \theta\left(\partial_{\zeta} \phi\right)^{2}
\end{gathered}
$$


It is deduced from (47) and (48) that

$$
\partial_{\xi} \phi \equiv 0, \quad \partial_{\zeta} \theta \equiv 0
$$

Using (50) and (45) in (46) gives the important equation

$$
\partial_{\eta} \partial_{\xi} \theta=-\left(\partial_{\xi} \theta\right)^{2}+c^{\prime} \sin \theta \partial_{\xi} \theta / c-c^{\prime \prime} \cos ^{2} \theta / c .
$$

Noting that $\partial_{\zeta} \phi$ is initially equal to $1 / \cos \theta_{i}=1 / x_{i}$, where $x_{i}$ is the initial $x$ coordinate of a ray, (49) may be integrated with the help of the first equation in (45) to give

$$
\partial_{\zeta} \phi=1 / x
$$

Thus (37) becomes

$$
\ln \left(I(\eta) / I\left(\eta_{0}\right)\right)=-\ln \left(x / x_{0}\right)-\int_{\eta_{0}}^{\eta} \partial_{\xi} \theta d \eta,
$$

where $x_{0}$ is the value of $x$ at $\eta_{0}$. Hence to evaluate the ray trajectory and intensity variation along a ray the non-trivial equations of (45) are integrated along with (51) and the last term in (53).

As an example, consider the case of propagation of a sound wave underwater in the sound velocity profile illustrated in Figure 5. The initial wavefront is considered to be spherical and of radius 1. A fourth order Runge-Kutta method has been used to carry out the integrations and a constant step size used. A key feature of this formulation is that the integrands are simply algebraic functions of the dependent variables, derivatives of the sound speed and elementary trigonometric 


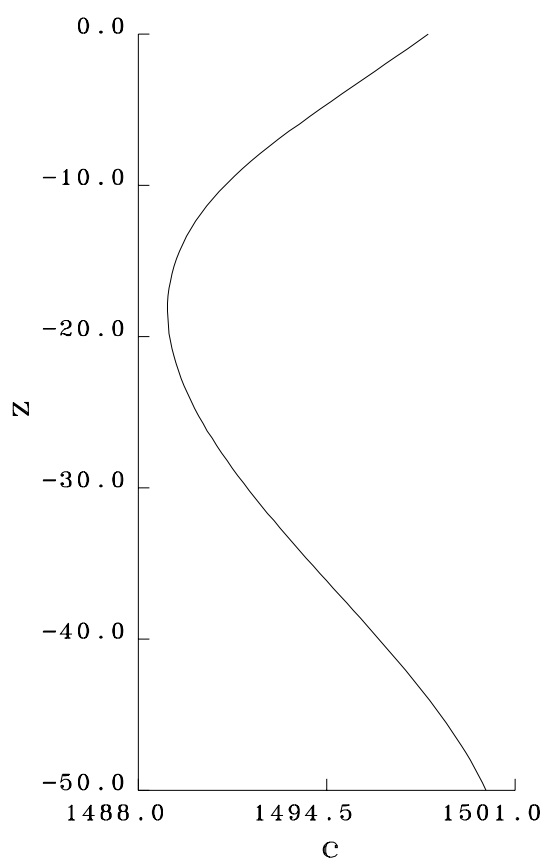

FIGURE 5. An example sound velocity profile for underwater sound propagation. 


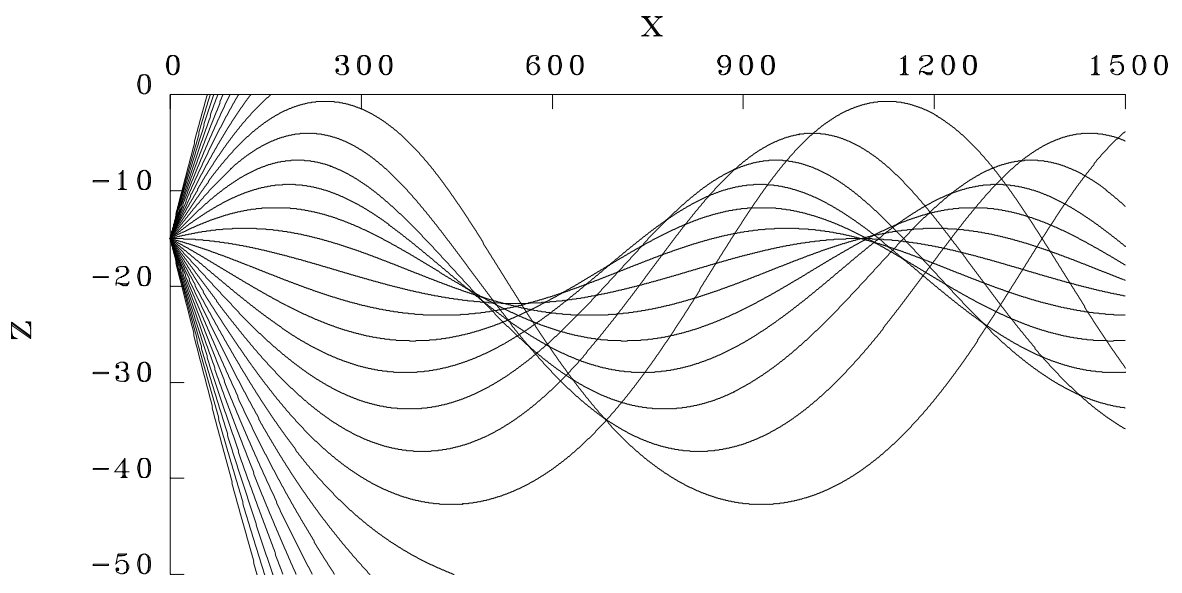

FIGURE 6. The trajectories of a collection of rays describing propagation in the sound velocity profile illustrated in Figure 5. The initial orientations of the rays lie between $-15^{\circ}$ and $15^{\circ}$. 
functions of $\theta$. This particularly facilitates calculation of ray trajectories which exhibit a waveguide-like behaviour as shown in Figure 6. This figure illustrates the trajectories of a collection of rays with $\theta_{i}$ lying between $\pm 15^{\circ}$ in $1^{\circ}$ increments. Whereas some formulations involving Cartesian coordinates yield ambiguities in the sign of the square root function at points where the ray changes direction from propagation downwards to upwards and vice-versa (see for example Whitham [8, p250]), the equations used here apply naturally and robustly along the whole of the ray. Indeed, as far as the equations for $x$ and $z$ are concerned, the magnitudes of the derivatives with respect to $\eta$ are always bounded by 1 . It is also remarked that in the event that adjacent rays cross, $\partial_{\xi} \theta$ becomes unbounded. Of course if such a circumstance occurs, ray acoustics is no longer appropriate for predicting the wave intensity. Note that this formulation is applicable even in the event that $c(z)$ has discontinuous derivatives at certain points, as may happen if this function is represented as piecewise linear. It merely requires that the discontinuities in $c^{\prime}$ and delta functions in $c^{\prime \prime}$ are appropriately dealt with in integration of the ray equations.

As a final remark on the usefulness of this formulation it is noted that boundaries such as the ocean surface or bottom may be dealt with in a number of ways. The propagation equations may be supplemented by appropriate reflection conditions. An alternative method is to consider that the boundaries are defined by thin regions of very high sound speed gradient, that in the limit of infinitesimal thickness yield the appropriate reflection behaviour. If the form of the ray trace equations presented here were numerically integrated in this case, a variable step size algorithm should be utilised since the propagation direction $\theta$ changes rapidly at reflection points. 
In conclusion, the mathematical formalism for acoustic ray tracing presented here is elegant with the coordinate system chosen so that it is defined by the motion of the wavefront itself. The coordinate system may thus be considered natural for this problem. The ease with which ray trajectories and intensity variation along a ray may be computed in non-homogeneous media demonstrates its practical usefulness.

\section{A Appendix}

Differentiation of (39) with respect to $\zeta$ and $\xi$ and conversion of derivatives of the sound speed using (11) yields

$$
\begin{aligned}
\partial_{\zeta} \partial_{\eta} \theta=- & \sin \theta\left[\sin \phi c_{x}-\cos \phi c_{y}\right] \partial_{\zeta} \phi / c \\
+ & {\left[\cos \phi \cos \theta c_{x}+\sin \phi \cos \theta c_{y}+\sin \theta c_{z}\right] \partial_{\zeta} \theta / c } \\
- & {\left[\cos \phi \sin \phi \sin \theta c_{x x}+\left(\sin ^{2} \phi-\cos ^{2} \phi\right) \sin \theta c_{x y}\right.} \\
& \left.-\cos \phi \sin \phi \sin \theta c_{y y}+\cos \phi \cos \theta c_{y z}-\sin \phi \cos \theta c_{z x}\right] / c \\
+ & {\left[\cos \phi \sin \phi \sin \theta c_{x}^{2}-\cos \phi \sin \phi \sin \theta c_{y}^{2}+\left(\sin ^{2} \phi\right.\right.} \\
& \left.\left.-\cos ^{2} \phi\right) \sin \theta c_{x} c_{y}+\cos \phi \cos \theta c_{y} c_{z}-\sin \phi \cos \theta c_{z} c_{x}\right] / c^{2} \\
\partial_{\zeta} \partial_{\eta} \phi= & {\left[\cos \phi c_{x}+\sin \phi c_{y}\right] \partial_{\zeta} \phi /(c \cos \theta) } \\
& +\sin \theta\left[\sin \phi c_{x}-\cos \phi c_{y}\right] \partial_{\zeta} \theta /\left(c \cos ^{2} \theta\right) \\
& -\left[\sin { }^{2} \phi c_{x x}+\cos ^{2} \phi c_{y y}-2 \sin \phi \cos \phi c_{x y}\right] /(c \cos \theta) \\
& +\left[\sin \phi c_{x}-\cos \phi c_{y}\right]^{2} /\left(c^{2} \cos \theta\right)
\end{aligned}
$$




$$
\begin{aligned}
\partial_{\xi} \partial_{\eta} \theta= & -\sin \theta\left[\sin \phi c_{x}-\cos \phi c_{y}\right] \partial_{\xi} \phi / c \\
+ & {\left[\cos \phi \cos \theta c_{x}+\sin \phi \cos \theta c_{y}+\sin \theta c_{z}\right] \partial_{\xi} \theta / c } \\
- & {\left[\cos ^{2} \phi \sin ^{2} \theta c_{x x}+\sin ^{2} \phi \sin ^{2} \theta c_{y y}\right.} \\
& +\cos ^{2} \theta c_{z z}+2 \cos \phi \sin \phi \sin ^{2} \theta c_{x y} \\
& \left.-2 \sin \phi \cos \theta \sin \theta c_{y z}-2 \cos \phi \cos \theta \sin \theta c_{z x}\right] / c \\
+ & {\left[\cos \phi \sin \theta c_{x}+\sin \phi \sin \theta c_{y}-\cos \theta c_{z}\right]^{2} / c^{2}, }
\end{aligned}
$$

$$
\begin{aligned}
\partial_{\xi} \partial_{\eta} \phi= & {\left[\cos \phi c_{x}+\sin \phi c_{y}\right] \partial_{\xi} \phi /(c \cos \theta) } \\
& +\sin \theta\left[\sin \phi c_{x}-\cos \phi c_{y}\right] \partial_{\xi} \theta /\left(c \cos ^{2} \theta\right) \\
& -\left[\cos \phi \sin \phi \sin \theta c_{x x}-\cos \phi \sin \phi \sin \theta c_{y y}+\left(\sin ^{2} \phi\right.\right. \\
& \left.\left.-\cos ^{2} \phi\right) \sin \theta c_{x y}+\cos \phi \cos \theta c_{y z}-\sin \phi \cos \theta c_{z x}\right] /(c \cos \theta) \\
& +\left[\cos \phi \sin \phi \sin \theta c_{x}^{2}-\cos \phi \sin \phi \sin \theta c_{y}^{2}\right. \\
& +\left(\sin ^{2} \phi-\cos ^{2} \phi\right) \sin \theta c_{x} c_{y} \\
& \left.+\cos \phi \cos \theta c_{y} c_{z}-\sin \phi \cos \theta c_{z} c_{x}\right] /\left(c^{2} \cos \theta\right) .
\end{aligned}
$$

\section{References}

[1] J. P. Best, "Accounting for transverse flow in the theory of geometrical shock dynamics", Proc. R. Soc. Lond. A 442 (1993) 585-598. 
[2] R. Buckley, "On the calculation of intensity in dispersive inhomogeneous media in the ray approximation", Proc. R. Soc. Lond. A 380 (1982) 201-209.

[3] K. G. Budden, "Ray tracing and the effect of ray divergence and convergence on signal intensity", Proc. R. Soc. Lond. A 432 (1991) 233-246.

[4] H. R. Krol, "Intensity calculations along a single ray", J. Acoust. Soc. Amer. 53 (1973) 864-868.

[5] L. P. Solomon \& L. Armijo, "An intensity differential equation in ray acoustics”, J. Acoust. Soc. Amer. 50 (1971) 960-963.

[6] P. Uginčius, "Intensity equations in ray acoustics. I", J. Acoust. Soc. Amer. 45 (1969) 193-205.

[7] P. Uginčius, "Intensity equations in ray acoustics. II", J. Acoust. Soc. Amer. 45 (1969) 206-209.

[8] G. B. Whitham, Linear and nonlinear waves (Wiley, New York, 1974) 